\section{Decrypting the role of Cripto in tumorigenesis}

\author{
Michael M. Shen
}

Center for Advanced Biotechnology and Medicine, and Department of Pediatrics, University of Medicine and Dentistry of New Jersey-Robert Wood Johnson Medical School, Piscataway, New Jersey, USA

The cell-surface associated molecule Cripto is overexpressed in a wide range of epithelial cancers, yet little is known about potential mechanisms by which Cripto expression might enhance tumorigenesis. A new study (see the related article beginning on page 575 ) reveals that binding of Cripto to the TGF- $\beta$ ligand Activin B can block Activin B-mediated suppression of cell proliferation. Furthermore, this study also demonstrates that antibody blockade of Cripto function may prove useful in the inhibition of tumorigenesis.

J. Clin. Invest. 112:500-502 (2003). doi:10.1172/JCI200319546.

Ever since the clinical success of the anti-HER-2 monoclonal antibody Herceptin in treating breast cancer, major efforts have been undertaken to identify cell-surface associated proteins that are associated with signal transduction pathways and that are upregulated in human cancers. The underlying rationale of this strategy has been that such proteins might play an essential role in maintenance of the transformed state, and that treatment with appropriate antibodies might thereby lead to inhibition of tumor growth, while leaving normal tissue relatively unaffected. In this issue of the JCI, Adkins and colleagues (1) provide convincing evidence that one such extracellular protein, Cripto, represents a promising therapeutic target that is upregulated in a wide range of epithelial cancers, including breast, stomach, and colon carcinomas.

\section{Cripto is a coreceptor for Nodal, a member of the TGF- $\beta$ family} Initially thought to be a secreted signaling molecule, Cripto is a small cysteine-rich protein that contains a single

\footnotetext{
Address correspondence to: Michael M. Shen, Center for Advanced Biotechnology and Medicine, 679 Hoes Lane, Piscataway, New Jersey 08854, USA.

Phone: (732) 235-5645; Fax: (732) 235-5373; E-mail: mshen@cabm.rutgers.edu.

Conflict of interest: The author has declared that no conflict of interest exists.

Nonstandard abbreviations used: Cripto, FRL-1, and Cryptic (CFC); glycosylphosphatidylinositol (GPI).
}

divergent EGF-like motif as well as a novel cysteine-rich domain termed the Cripto, FRL-1, and Cryptic (CFC) motif, and is attached to the cell membrane through a glycosyl-phosphatidylinosital (GPI) linkage. When it was originally cloned fifteen years ago as an accidental fusion transcript in a cDNA library screen, it was named Cripto for its mysterious lack of relationship to known proteins and signaling pathways (2). Several years ago, however, it became apparent that Cripto was the founding member of the EGF-CFC gene family, which is conserved among vertebrates, with homologs in chick, frogs, and zebrafish (3). Molecular genetic studies in fish and mice have revealed that EGF-CFC proteins play essential roles in early embryonic development in specification of the anterior-posterior and left-right body axes, as well as in formation of the primary germ layers during gastrulation $(3,4)$.

Further studies have demonstrated that EGF-CFC proteins act as coreceptors for Nodal, a member of the TGF- $\beta$ superfamily. In particular, membranebound Cripto appears to recruit Nodal to an activin receptor complex composed of a dimer of the type I serinethreonine receptor ActRIB (also known as ALK4) and a dimeric type II activin receptor, either ActRII or ActRIIB. Following receptor activation, Smad2 and/or Smad3 are phosphorylated and accumulate together with Smad4 in the nucleus to mediate transcriptional responses $(5,6)$. Interestingly, the interaction of Cripto with ActRIB requires its CFC motif, whereas Cripto binding to Nodal utilizes the EGF motif and requires post-translational modification by $O$-fucosylation, an unusual form of glycosylation found on certain EGF motif-containing proteins (5-7). In the absence of EGF-CFC proteins, Nodal lacks signaling activity through activin receptors, in contrast to Activin, which does not appear to require a coreceptor for its signaling activity (Figure 1, a and b) $(6,8)$.

However, these previous studies have left unresolved the significance of the widespread overexpression of Cripto in human epithelial cancers. Many early studies have shown that Cripto is highly overexpressed in breast, pancreatic, ovarian, and colon carcinomas, but the functional significance of these findings, if any, has remained elusive (9). Is Cripto activity essential for tumor growth and/or maintenance, and furthermore, does Cripto overexpression mediate signaling by Nodal in cancer, or is it independent of Nodal activity? Suggestive evidence regarding the former question has been provided by studies showing that Cripto expression can partially transform certain mammary cell lines (10) and that antisense inhibition of Cripto can lead to loss of the transformed phenotype of colon carcinoma cells (11).

\section{A new role for Cripto as an inhibitor of Activin signaling}

A new explanation for the functional significance of Cripto overexpression in epithelial cancers has now emerged from the work of Adkins et al. (1), as well as of Gray et al. (12). Both of these studies demonstrate that Activin signaling can be blocked by Cripto overexpression in a variety of cell culture assays, a hitherto unsuspected activity for EGF-CFC proteins. At a biochemical level, this inhibitory activity of Cripto results in inability to form an Activin/ActRII/ActRIB signaling complex. Furthermore, Adkins and colleagues demonstrate the ability of specific Cripto monoclonal antibodies to inhibit tumor cell proliferation in two distinct xenograft models. Using a large panel of Cripto 

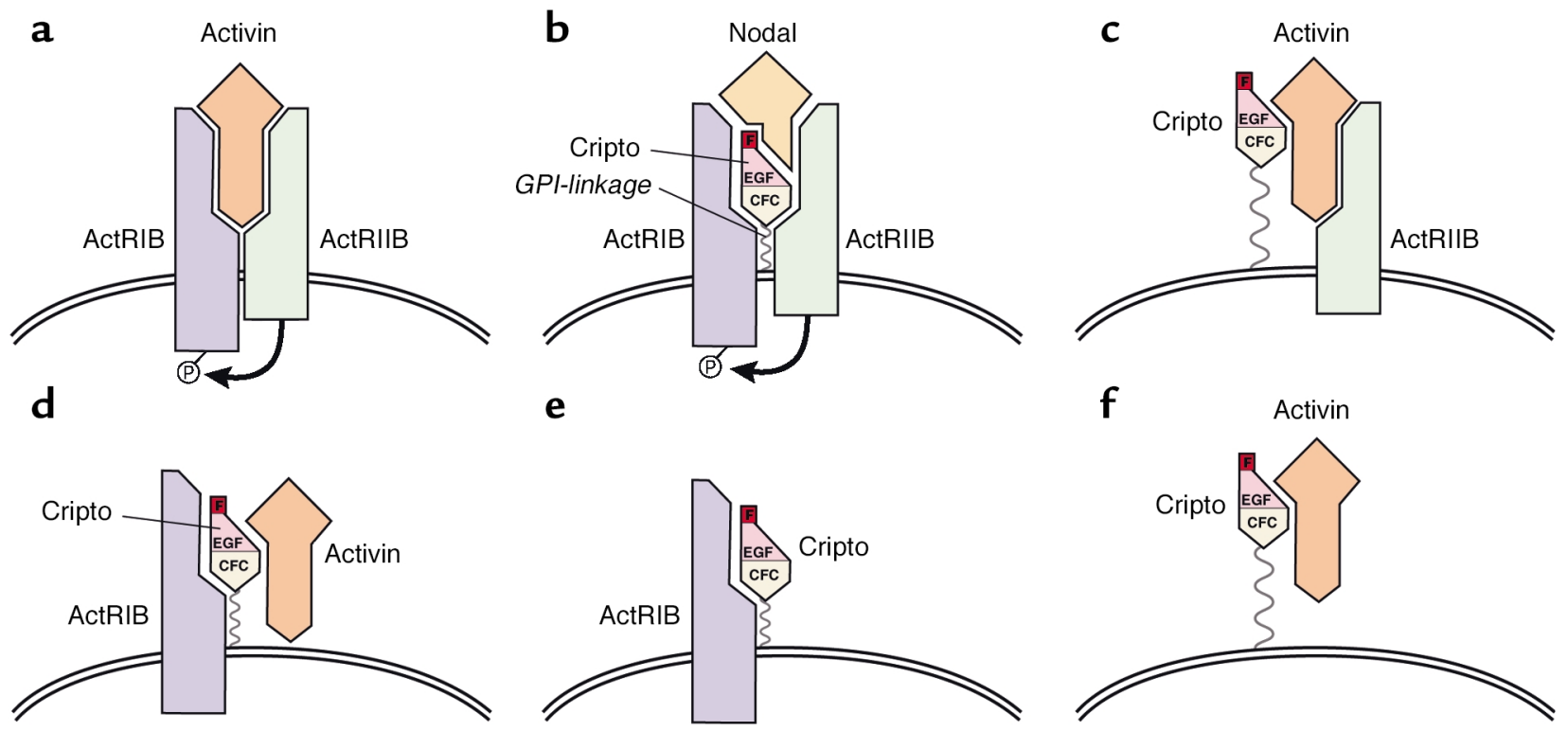

Figure 1

Schematic models for the role of Cripto in Activin and Nodal signaling. For simplicity, TGF- $\beta$ ligands and receptors are represented as monomers. (a) Activin signaling utilizes ActRIB and ActRII or ActRIIB, resulting in an active receptor complex and phosphorylation (P) of the type I receptor. (b) Nodal signaling requires Cripto, which is GPI-linked, in addition to ActRIB and ActRIIB. Nodal interacts with Cripto through the EGF motif, and requires the presence of an $O$-fucose modification (red F) on Cripto. (c-f) Four possible inactive complexes resulting in inhibition of Activin signaling by Cripto. Gray and colleagues (12) propose an inactive Cripto/Activin/ActRII complex (c) and do not detect direct interaction between Cripto and Activin (f). In contrast, Adkins and colleagues (1) propose three potential inactive complexes that do not include ActRII (d-f).

monoclonals, they find that the ability of Cripto antibodies to inhibit tumor growth is correlated with their ability to inhibit Activin signaling, indicating that their efficacy requires inactivation of Cripto function, and is not simply a consequence of antibody binding. These new findings raise the interesting possibility that Cripto overexpression may play an early role in cancer progression through inhibition of the tumorsuppressing effects of Activin.

However, these studies disagree on the key point of whether Activin can bind directly to Cripto, or whether their interaction is mediated by activin receptors. Gray and colleagues show that Cripto can inhibit Activin A (a homodimer of Activin $\beta$ A subunits) in cell culture activity assays, but they are unable to detect direct interaction of Activin A with Cripto in the absence of activin receptors; they note similar findings for Activin $B$ (a homodimer of Activin $\beta B$ subunits) (12). However, they find that Activin A can interact with Cripto in the presence of type II activin receptors, and conclude that Cripto can inhibit binding of Activin A to ActRIB through formation of an inactive Activin/ActRII/Cripto complex (Figure 1c). This model is analogous to that from a previous study by Lewis and colleagues, which showed that Inhibin (a heterodimer of Inhibin $\alpha$ and Activin $\alpha$ or $\beta$ subunits) interacts with betaglycan and ActRII to block Activin signaling (13). In contrast with these findings, the study by Adkins and colleagues demonstrates a direct protein interaction between soluble Cripto and Activin B in vitro, with an apparent affinity of $1 \mathrm{nM}$, but fails to detect any interaction between Cripto and Activin A or any inhibitory activity of Cripto for Activin A in cell culture assays (1).

Another discrepancy concerns whether Nodal and Activin interact with similar regions of the Cripto protein, an inconsistency that has implications for the molecular mechanism by which Cripto inhibits Activin function (Figure 1, c-f). Gray and colleagues show that interaction of Activin A with Cripto requires an intact EGF motif, and present cell culture data suggesting that Cripto can simulta- neously potentiate Nodal signaling and inhibit Activin signaling, supporting a model in which Nodal and Activin compete for binding to similar sites on Cripto (12). In contrast, Adkins and colleagues show that a CFC-specific monoclonal blocks interaction of Cripto with Activin B, suggesting that Activin B and Nodal bind to distinct regions of Cripto. This conclusion is further supported by the finding that a Cripto protein mutated at the $O$-fucose addition site within the EGF motif can still interact with Activin B but not with Nodal, suggesting that differential glycosylation may regulate the interaction of Cripto with distinct TGF- $\beta$ ligands in vivo.

\section{Does Cripto inhibit Activin signaling during tumorigenesis?}

The studies of Adkins and colleagues do not yet resolve whether the physiological mode of action of the Cripto antibodies in xenograft assays is due to inhibition of endogenous Activin activity. One likely alternative is that Cripto may be acting as a coreceptor for Nodal, 
which is unexpectedly expressed at low levels in normal mammary glands and in a wide range of tumor cell lines $(1,14)$. Another possibility is that Cripto may be mediating the function of other TGF- $\beta$ family ligands, supported by the recent report that Cripto can mediate signaling by frog Vg1 and its mammalian homolog GDF1 (15). Finally, Cripto has been suggested to act as a signaling factor that can activate Akt and MAPK pathways independently of activin receptor and TGF- $\beta$ signaling $(16,17)$. Nonetheless, the observation that a Cripto antibody that binds to the EGF motif and blocks Nodal binding is significantly less effective in xenograft assays than an antibody that binds the CFC motif and blocks Cripto interaction with ActRIB argues that Cripto inhibition of Activin signaling is at least an important component of its role in tumor growth and/or maintenance (1).

Indeed, it is conceivable that Cripto could promote tumorigenesis both by inhibiting the tumor suppressor activity of Activin and by mediating signaling by Nodal or related TGF- $\beta$ ligands. Moreover, these potential functions of Cripto might be temporally unrelated, since TGF- $\beta$ signaling is known to play dual opposing roles during cancer progression. Thus, early in progression, TGF- $\beta$ signaling inhibits cellular proliferation, thereby acting as a tumor suppressor, while later in cancer progression, TGF- $\beta$ signaling seems to promote metastasis, perhaps through mediating an epithelial-mesenchymal transition (18). Consequently, loss-offunction analyses using in vivo systems and conditional temporal inactivation approaches may be necessary to distinguish between these models for Cripto function during cancer progression.

The exciting finding that antibody blockade of Cripto has a strong effect in xenograft models indicates that Cripto functions in a central pathway for cell proliferation and/or maintenance of the transformed state. While much more analysis is needed to decode the molecular mechanisms of Cripto function, the work of Adkins et al. (1) and Gray et al. (12) now provides a glimmer of understanding as to the functions of this enigmatic protein in tumorigenesis.

\section{Acknowledgements}

Michael M. Shen is supported by grants from the NIH.

1. Adkins, H.B., et al. 2003. Antibody blockade of the Cripto CFC domain suppresses tumor cell growth in vivo. J. Clin. Invest. 112:575-587. doi:10.1172/JCI200317788.

2. Ciccodicola, A., et al. 1989. Molecular characterization of a gene of the "EGF family" expressed in undifferentiated human NTERA2 teratocarcinoma cells. EMBO J. 8:1987-1991.

3. Shen, M.M., and Schier, A.F. 2000. The EGF-CFC gene family in vertebrate development. Trends Genet. 16:303-309.

4. Whitman, M. 2001. Nodal signaling in early vertebrate embryos. Themes and variations. Dev Cell. 1:605-617.
5. Yeo, C., and Whitman, M. 2001. Nodal signals to Smads through Cripto-dependent and Criptoindependent mechanisms. Mol. Cell. 7:949-957.

6. Yan, Y.T., et al. 2002. Dual roles of Cripto as a ligand and coreceptor in the nodal signaling pathway. Mol. Cell. Biol. 22:4439-4449.

7. Schiffer, S.G., et al. 2001. Fucosylation of Cripto is required for its ability to facilitate nodal signaling. J. Biol. Chem. 276:37769-37778.

8. Kumar, A., et al. 2001. Nodal signaling uses activin and transforming growth factorbeta receptor-regulated Smads. J. Biol. Chem. 276:656-661

9. Salomon, D.S., et al. 2000. The EGF-CFC family: novel epidermal growth factor-related proteins in development and cancer. Endocr. Relat. Cancer. 7:199-226.

10. Ciardiello, F., Dono, R., Kim, N., Persico, M.G., and Salomon, D.S. 1991. Expression of cripto, a novel gene of the epidermal growth factor gene family, leads to in vitro transformation of a normal mouse mammary epithelial cell line. Cancer Res. 51:1051-1054.

11. Ciardiello, F., et al. 1994. Inhibition of CRIPTO expression and tumorigenicity in human colon cancer cells by antisense RNA and oligodeoxynucleotides. Oncogene. 9:291-298.

12. Gray, P.C., Harrison, C.A., and Vale, W. 2003. Cripto forms a complex with activin and type II activin receptors and can block activin signaling. Proc. Natl. Acad. Sci. U. S. A. 100:5193-5198.

13. Lewis, K.A., et al. 2000. Betaglycan binds inhibin and can mediate functional antagonism of activin signaling. Nature. 404:411-414.

14. Bianco, C., et al. 2002. Cripto-1 activates nodaland ALK4-dependent and -independent signaling pathways in mammary epithelial cells. $\mathrm{Mol}$. Cell Biol. 22:2586-2597.

15. Cheng, S.K., Olale, F., Bennett, J.T., Brivanlou, A.H., and Schier, A.F. 2003. EGF-CFC proteins are essential coreceptors for the TGF-beta signals Vg1 and GDF1. Genes Dev. 17:31-36.

16. Ebert, A.D., et al. 1999. Cripto-1 induces phosphatidylinositol 3'-kinase-dependent phosphorylation of AKT and glycogen synthase kinase $3 \beta$ in human cervical carcinoma cells. Cancer Res. 59:4502-4505.

17. Bianco, C., et al. 2003. A Nodal- and ALK4-independent signaling pathway activated by Cripto1 through Glypican-1 and c-Src. Cancer Res. 63:1192-1197.

18. Derynck, R., Akhurst, R.J., and Balmain, A. 2001. TGF-beta signaling in tumor suppression and cancer progression. Nat. Genet. 29:117-129. 